Published in final edited form as:

Comput Aided Surg. 2006 May ; 11(3): 119-125.

\title{
Respiratory motion compensation with tracked internal and external sensors during CT-guided procedures
}

\author{
J. BORGERT ${ }^{1}$, S. KRÜGER ${ }^{1}$, H. TIMINGER ${ }^{1}$, J. KRÜCKER ${ }^{2}$, N. GLOSSOP $^{3}$, A. DURRANI $^{4}$, \\ A. VISWANATHAN ${ }^{4}$, and B.J. WOOD ${ }^{4}$ \\ 1 Philips Research Laboratories, Hamburg, Germany
}

2Philips Research USA, Briarcliff Manor, New York, USA

3Traxtal, Inc., Bellaire, Texas, USA

4Diagnostic Radiology Department, National Institutes of Health, Bethesda, Maryland, USA

\begin{abstract}
This paper investigates the possibility of using the motion of a patient's anterior surface in combination with a motion model to compensate for internal respiratory motion during tracked biopsies. Position data from two electromagnetically tracked sensors, one placed on the patient's sternum, the other incorporated into a biopsy needle, were acquired during a liver biopsy. The data were used to evaluate the correlation between the position measurements of the two sensors and to derive an affine motion model to assess respiratory motion compensation for image-guided interventional procedures. The correlation reached up to $94 \%$ for ranges of steady respiration. The residual motion of the internal sensor after compensation is reduced by a factor of approximately four.
\end{abstract}

\section{Keywords}

Respiratory motion compensation; interventional navigation; electromagnetic tracking

\section{Introduction}

Computer aided surgery and interventional procedures using magnetic tracking systems for image-guidance have recently shown promising results [1-5]. The magnetic tracking system (MTS) is used to determine the position and orientation of interventional devices such as biopsy or radiofrequency ablation (RFA) needles. The devices are registered to pre-procedurally acquired anatomical images, usually from computed tomography (CT) or magnetic resonance imaging (MRI), to enable image guidance during the application.

Biopsies and ablations have become standard techniques for the diagnosis and treatment of certain oncology applications, including lung, kidney, and liver tumors [6]. These procedures are usually performed under ultrasound, CT, CT-fluoroscopy, or even MRI guidance.

Procedures that target lesions located in the liver are generally performed percutaneously or with laparoscopic support [7], reducing the surgical exposure required [8]. In contrast to wellestablished optical position measurement systems, magnetic tracking systems do not suffer

Correspondence: Jörn Borgert, Ph.D., Senior Scientist, Philips Research Laboratories, P.O. Box 63 05 65, Röntgenstrasse 24-26, D-22315 Hamburg, Germany. E-mail: joern.borgert@philips.com.

Part of this research was previously presented at the 19th International Congress and Exhibition on Computer Assisted Radiology and Surgery (CARS 2005), held in Berlin, Germany, in June 2005. 
from line-of-sight restrictions and are therefore especially suited for use in percutaneous procedures, since the tracked element can be placed at the tip of a flexible instrument. The potential advantages of these less invasive procedures include the ability to be used in an outpatient setting, less blood loss, faster recovery, shorter hospital stays, reduced use of pain medication, and reduced costs. In addition, some interventional or minimally invasive procedures can be performed on patients who are unable to undergo traditional surgical intervention due to advanced age, extent of disease, or inaccessibility of the lesion. For these patients in particular, alternatives are limited.

A major challenge for navigation during image-guided minimally invasive procedures has been the accurate characterization of and compensation for respiratory motion. A normal breathing cycle may, for example, change the position of tumors located in the lung by approximately $1-3 \mathrm{~cm}$ [9], whereas abdominal organ movement can amount to $1.5-6 \mathrm{~cm}$ in the cranial-caudal direction, predominantly as a result of diaphragmatic motion [10,11]. Various techniques have been devised to better target such lesions.

Simple approaches try to optimize the use of standard equipment and resources. The patient is positioned so as to allow easy access to the target region. This is coupled with the timing of a contrast agent injection to offer the clinician the opportunity for improved lesion detection on pre-procedural images and successful targeting of difficult lesions [12]. Using CT imaging in fluoroscopy mode allows for a nearly real-time visualization of the needle placement and reduces the total scan time [13-15]. Unfortunately, a well-known drawback of CT-fluoroscopy during interventions is inadvertent exposure of the physician and patient to the primary beam [16].

Other approaches use additional instrumentation to characterize and potentially compensate for respiratory motion that instigates lesion movement. Simple respiration monitoring devices have been developed to enable physicians to receive feedback on the respiratory status during successive CT scans. Such devices use a lever mechanism equipped with a ball that deflects with each inhalation. The motion of the ball undergoes transduction into a voltage signal that can be provided to the patient as audio or visual feedback [17]. Other devices use spirometric measurement of airflow and strain gauge determination of abdominal circumference [18-20]. The use of an uncomfortable mouthpiece is a drawback of the spirometric technique, and the instruments for the strain gauge technique are more complicated to handle than devices for breath-hold feedback.

Other techniques like gating have the potential to minimize respiration-induced target motion during image acquisition and device tracking. Apart from the devices for respiratory control mentioned above, a gating system can, for example, comprise a reflective marker placed on the patient's anterior surface and tracked with a camera. Suitable intervals can be defined to limit the motion of the diaphragm during image acquisition to less than $1 \mathrm{~cm}$ while otherwise allowing for free breathing [21]. Work in the area of compensation for cardiac motion, which in a simple model consists of motion due to respiration and heartbeat, uses downstream respiratory motion compensation with an affine motion model [22] to relate position measurements of interventional devices, e.g., guidewires and catheters, to pre-procedurally acquired images. Another area where motion characterization and compensation lead to improved results is intensity-modulated radiation therapy [23].

In the end, successful compensation for respiratory motion is essential to improve registration to pre-procedural images and to enable navigation in the presence of patient motion due to respiration. This paper investigates the possibility of using an externally placed electromagnetic sensor in combination with a motion model to compensate for internal respiratory motion during tracked biopsies. An additional electromagnetically tracked sternum- 
mounted sensor was tested clinically as a method of motion acquisition during an electromagnetically tracked biopsy. The data were analyzed with respect to the correlation of the motion of the external and internal sensor and the possibility of deploying a motion model to compensate for the motion of the internal sensor due to respiration.

\section{Materials and methods}

\section{Clinical setup}

The raw data for the study were acquired during a tracked biopsy of a $15 \mathrm{~mm}$ hepatocellular carcinoma. The biopsy was performed on a sedated but otherwise conscious, unventilated 61year-old male patient. The study was approved by the hospital's Institutional Review Board (IRB), and the patient signed written informed consent.

The respiratory cycle length varied between 3 and 5.5 seconds, depending on the patient's state of sedation. Trackable needles with 5 degrees of freedom (DOF) and internal sensor coils embedded in the stylet tip were designed, tested, sterilized, and used for the procedure. An additional 6-DOF sensor was placed on the patient's sternum. The needles were connected to an electromagnetic tracking system (NDI Aurora, Northern Digital Inc., Waterloo, Ontario, Canada), which was integrated into a custom-made tracking and visualization workstation prototype for interventional navigation. The system was used to acquire and log the position and orientation of the connected devices. For the current analysis, only position data were evaluated.

Data acquisition was performed with a mean rate of 32.5 samples per second for selected parts of the whole intervention, totaling $720 \mathrm{~s}$ of tracking data. During parts of this acquisition, the needle was advanced and repositioned in the liver. Two ranges of data with steady respiration, consecutively named datal and data2, were selected for a detailed analysis and assessment of the motion model. One of the ranges included a needle manipulation (see Figures 1 and 2) to assess the interplay between this movement and the motion model. Table I lists characteristics of the data.

\section{Correlation matrix analysis}

The correlation of the motion of the needle placed in the liver with the sternum sensor was determined by correlation matrix analysis. The entries of the correlation matrix cor $_{m n}$ are defined by the ratio of the entries of the covariance matrix $\operatorname{cov}_{m n}$ and the respective product of the standard deviations $\sigma_{m}$ and $\sigma_{n}$ :

$$
\operatorname{cor}_{m n}=\frac{\operatorname{cov}_{m n}}{\sigma_{m} \sigma_{n}}=\frac{\langle(m-\langle m\rangle)(n-\langle n\rangle)\rangle}{\sigma_{m} \sigma_{n}}
$$

with $m$ and $n$ running over all individual coordinates, i.e., $m, n \in\left\{x, y, z, x^{\prime}, y^{\prime}, z^{\prime}\right\}$, and the square brackets denoting the mean over the set of samples under examination, for example, $\langle x\rangle=1 / N \sum_{i=1}^{N} x_{i}$ for $m=x$ and a total of $N$ samples. For the remainder of the text, the unprimed coordinates represent the coordinates of the liver or internal sensor, whereas the primed coordinates represent the coordinates of the sternum or external sensor placed on the patient's body. So, for the $x$-coordinate from one sensor and the $x$ '-coordinate from the other sensor, the correlation yields

$$
\begin{aligned}
\operatorname{cor}_{x x^{\prime}} & =\frac{\operatorname{cov}_{x x^{\prime}}}{\sigma_{x} \sigma_{\gamma^{\prime}}} \\
& =\frac{\left\langle(x-\langle x\rangle)\left(x^{\prime}-\left\langle x^{\prime}\right\rangle\right)\right\rangle}{\sigma_{x} \sigma_{x^{\prime}}} \\
& =\frac{1}{\sigma_{x} \sigma_{\sigma^{\prime}}} \frac{1}{N} \\
& =\sum_{i=1}^{N}\left[\left(x_{i}-\frac{1}{N} \sum_{j=1}^{N} x_{j}\right) \cdot\left(x_{i}^{\prime}-\frac{1}{N} \sum_{j=1}^{N} x_{j}^{\prime}\right)\right]
\end{aligned}
$$


and

$$
\begin{aligned}
\operatorname{cor}_{x x^{\prime}}= & \frac{1}{N} \sum_{i=1}^{N}\left[\left(x_{i}-\frac{1}{N} \sum_{j=1}^{N} x_{j}\right)\right. \\
& \left.\times\left(x_{i}^{\prime}-\frac{1}{N} \sum_{j=1}^{N} x_{j}^{\prime}\right)\right] \\
& \times\left[\frac{1}{N} \sum_{j=1}^{N}\left(x_{i}-\frac{1}{N} \sum_{j=1}^{N} x_{j}\right)^{2}\right]^{1 / 2} \\
& \times\left[\frac{1}{N} \sum_{i=1}^{N}\left(x_{i}^{\prime}-\frac{1}{N} \sum_{j=1}^{N} x_{j}^{\prime}\right)^{2}\right]^{1 / 2}
\end{aligned}
$$

with the usual definition of the standard deviation.

Specific entries of the resulting $6 \times 6$ correlation matrix from all 6 coordinates

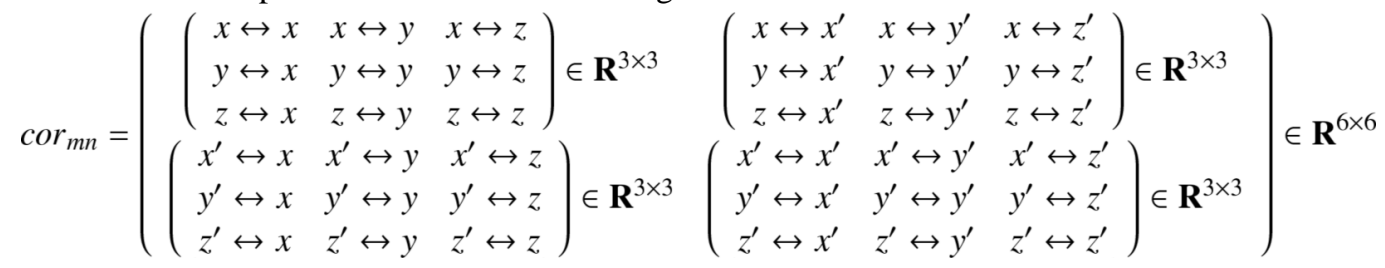

$$
\begin{aligned}
& \operatorname{cor}_{m n}=\left(\begin{array}{cc}
\operatorname{cor}_{s t} \in 3 \times 3 & \operatorname{cor}_{s t^{\prime}} \in 3 \times 3 \\
\operatorname{cor}_{s^{\prime} t} \in 3 \times 3 & \operatorname{cor}_{s^{\prime} t^{\prime}} \in 3 \times 3
\end{array}\right) \in \mathbf{R}^{6 \times 6}
\end{aligned}
$$

provide information on the correlation between the coordinates $(x, y, z)$ or $\left(x^{\prime}, y^{\prime}, z^{\prime}\right)$ of an individual sensor, $\operatorname{cor}_{s t}$ or $\operatorname{cor}_{s^{\prime}} t^{\prime}$, as well as on the correlation between coordinates from the two different sensors, $c o r_{s t^{\prime}}$ or $c o r_{s^{\prime} t}$. Of course, $c o r_{s t^{\prime}}=c o r_{s^{\prime} t}^{T}$ holds true.

The sum over the absolute values of the entries of the submatrix for the correlation of the two different sensors is used as a measure to quantify their correlation. The individual entries range from -1 for complete anti-correlation to +1 for complete correlation, whereas a value of 0 represents no correlation at all. For a $3 \times 3$ matrix, the number of entries is 9 , so the percent correlation for sensor-to-sensor correlation is given by

$100 \% \cdot \frac{1}{9} \cdot$ sum over absolute values of entries of $\left(\begin{array}{cccc}x \leftrightarrow x^{\prime} & x \leftrightarrow y^{\prime} & x \leftrightarrow z^{\prime} \\ y \leftrightarrow x^{\prime} & y \leftrightarrow y^{\prime} & y \leftrightarrow z^{\prime} \\ z \leftrightarrow x^{\prime} & z \leftrightarrow y^{\prime} & z \leftrightarrow z^{\prime}\end{array}\right)=100 \% \cdot \frac{1}{9}\left(\sum_{\substack{s \in\{x, y, z\} \\ t^{\prime} \in\left\{x^{\prime}, y^{\prime}, z^{\prime}\right\}}}\left|\operatorname{cor}_{s t^{\prime}}\right|\right.$.

The determination of the correlation as a function of time was performed using a sliding window containing 150 samples, covering approximately 4.6 seconds of data, to determine the integral correlation for approximately 1 to 1.5 respiratory cycles (cf. Figure 1 ). The mean and standard deviation of the correlation values in the sliding window were computed. This correlation analysis was performed for all of the available data, as well as for the selected ranges of steady respiration, datal and data2.

\section{Affine motion model}

To obtain an impression of the potential for compensating for the displacement of the internal sensor due to respiration, an affine motion model was derived using the parts of the data exhibiting steady respiration (datal and data2). When the needle is not advanced or retracted, the coordinates obtained from the position measurements should be constant, with any residual 
displacement being due to respiration, assuming no needle slippage. The position measurements from the sternum sensor and the related measurements from the liver sensor from a limited amount of data (marked learning range in Figure 1) were used to derive a model for respiratory motion compensation. Applying this model to the data, the residual displacement compared to the displacement without compensation is a measure of the effectiveness of the motion model and motion compensation.

To compensate an acquired position measurement of the liver sensor, its displacement from a given reference position due to respiratory motion is corrected by the respective displacement measured by the sternum sensor. Therefore, the displacement of the sternum sensor is transformed by an affine transformation to derive the appropriate correction for the liver sensor. The actual generalized affine matrix consists of 12 free parameters

$$
A=\left(\begin{array}{cccc}
a_{11} & a_{12} & a_{13} & a_{14} \\
a_{21} & a_{22} & a_{23} & a_{24} \\
a_{31} & a_{32} & a_{33} & a_{34} \\
0 & 0 & 0 & 1
\end{array}\right)
$$

and represents the transformation of the displacement of the sternum sensor to the displacement of the liver sensor in homogeneous coordinates. The unknown entries of $A$ are determined by finding those $a_{i j}$ that minimize

$$
\sum\left(A \bullet\left(x_{\text {disp }}^{\prime}, y_{\text {disp }}^{\prime}, z_{\text {disp }}^{\prime}, 1\right)-\left(x_{\text {disp }}, y_{\text {disp }}, z_{\text {disp }}, 1\right)\right)^{2},
$$

where

$$
\begin{gathered}
x_{d i s p}^{\prime}=x^{\prime}-\frac{1}{N} \sum_{i=1}^{N} x_{i}^{\prime}, \\
y_{\text {disp }}=y-\frac{1}{N} \sum_{i=1}^{N} y_{i}, \quad \text { and so forth } \cdots
\end{gathered}
$$

is the displacement from the individual sensor's reference position. In this particular case, the reference position was chosen to be the mean position of the liver sensor over time. This ensures independence from any choice of reference frame, e.g., the coordinate system of the tracking system or that of the CT system, and registration. It is, however, sufficient to measure the efficiency of a potential compensation. More realistically, the individual sensor positions would be referenced to the respiratory phase during image acquisition, which enables the relation of the liver sensors' position to previously acquired image data.

Given an actual measurement of the internal sensor $(x, y, z)$, the compensated measurement in homogeneous coordinates is derived by

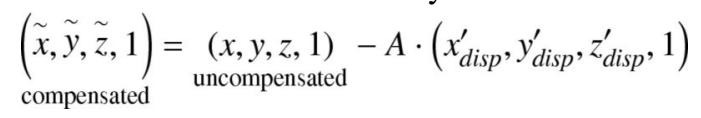

and

$$
\underset{\text { compensated }}{\left(\tilde{x}_{\text {disp }}, \tilde{y}_{\text {disp }}, \tilde{z}_{\text {disp }}\right)}=\underset{\text { compensated }}{(\tilde{x}, \tilde{y}, \tilde{z})}-\left(\frac{1}{N} \sum_{i=1}^{N} x_{i}, \frac{1}{N} \sum_{i=1}^{N} y_{i}, \frac{1}{N} \sum_{i=1}^{N} z_{i}\right) .
$$

To measure the efficiency of the compensation, the affine model built from a subset of 200 samples (learning range: $\sim 6 \mathrm{~s}$ ) of datal and data2, as shown in Figure 1, was used to compensate all of the respective samples of datal and data2. In the data 2 case, the evaluation 
of the efficiency of the compensation was performed for the first $23 \mathrm{~s}$ and the last $15 \mathrm{~s}$ separately, to assess how the compensation interplays with needle manipulations. The efficiency is given by a mean residual displacement after compensation together with a standard deviation.

\section{Results}

The overall distance between the liver sensor (intra-corporal) and the sternum sensor was 196.5 $\pm 9.1 \mathrm{~mm}$ during data acquisition.

The correlation between the motion of the sensor of the needle placed in the suspected carcinoma and the motion of the sensor on the sternum was $77.51 \pm 19.44 \%$ for all available data $(720 \mathrm{~s})$. The correlation for the selected ranges of steady respiration showed a value of $90.54 \pm 7.80 \%$ for datal and $94.61 \pm 5.63 \%$ for data2. Figure 1 shows the absolute values of the respective position measurements (in arbitrary scaling) together with the sliding window correlation in percent for datal and data2.

Without motion compensation, the mean residual displacement from the mean position of the liver sensor amounts to $3.90 \pm 2.00 \mathrm{~mm}$ for datal and $4.18 \pm 2.10 \mathrm{~mm}$ for data2, which already represent good conditions compared to the organ motion reported in references 9-11. After compensation, an improvement of the residual displacement to $0.99 \pm 0.66 \mathrm{~mm}$ for datal (a factor of $\sim 4$ ) and $0.94 \pm 0.46 \mathrm{~mm}$ for the first $23 \mathrm{~s}$ of data2 (also a factor of $\sim 4$ ) could be realized, as shown in Table II. The last $15 \mathrm{~s}$ of data2 resulted in a residual displacement of 3.18 $\pm 0.48 \mathrm{~mm}$, which clearly reflected the needle's movement of approximately $3 \mathrm{~mm}$. Subtracting this extra displacement from the residual displacement leads to results comparable to those in the case without manipulation. Figure 2 (bottom) shows the influence of the needle manipulation on the compensation and residual displacement, which clearly reflects the needle's movement. The reduction in standard deviation from the uncompensated case to the compensated case amounts to an improvement by a factor of $\sim 4$ in all cases, including the case after needle manipulation.

The results for the compensation of datal and data 2 can be compared with a compensation using a free-form deformation model as described in references 24 and 25, deploying radial basis functions [26] for interpolation. This compensation resulted in a residual displacement of $1.04 \pm 0.91 \mathrm{~mm}$ for datal and $1.99 \pm 1.09 \mathrm{~mm}$ for data 2 .

\section{Conclusions}

An additional external electromagnetically tracked sternum sensor was used to set up an affine respiratory motion model during CT-guided interventions. The motion model was used to compensate for the respiratory motion of a sensor introduced into an inner organ. It was demonstrated that the motion of the additional sensor placed on the patient's sternum showed a high correlation with the internal sensor. The overall correlation without pre-selection of data is already high (nearly 78\%), although it contains all types of motion artifacts, including, for example, advancing or repositioning the needle, as well as organ shift caused by overall patient motion.

For the ranges of steady respiration with or without only minimal needle or patient motion, the correlation reaches up to $94 \%$ for several respiratory cycles.

An affine motion model driven by the motion data of the sternum sensor reduced the residual displacement of the internal sensor by a factor of approximately 4 . Furthermore, it was possible to qualitatively and quantitatively detect the manipulation of the needle present in the data. 
Given the high correlation, it can be expected that the combination of internally tracked sensors with additional external sensors on the patient's sternum can be used as a reliable means of respiratory motion detection and compensation. The general applicability of this finding will have to be proven in further studies, together with the assessment of more sophisticated models for motion compensation. Such models could include elastic deformable dynamic models to account for tissue warping, such as may occur with applied torque during needle manipulations.

The potential correction of respiratory misregistration with a compensation model will lay out a course for successful integration and clinical application of electromagnetic tracking of medical devices. Image guidance will play an important role in effective, as well as conservative, cancer treatment in what it is hoped will be the near future.

\section{Acknowledgements and disclosure}

This study was supported in part by intramural research funds of the NIH Clinical Center. Philips, BJW, NIH, NG and Traxtal Technologies have related intellectual property. NIH and Philips Medical Systems have a Collaborative Research and Development Agreement.

\section{References}

1. Wood BJ, Zhang H, Durrani A, Glossop N, Ranjan S, Lindisch D, Levy E, Banovac F, Borgert J, Krüger S, Krücker J, Viswanathan A, Cleary K. Navigation with electromagnetic tracking for interventional radiology procedures: A feasibility study. J Vasc Interv Radiol 2005;16:493-505. [PubMed: 15802449]

2. Solomon SB, Dickfield T, Calkins H. Real-time cardiac catheter navigation on three-dimensional CT images. J Interventional Cardiac Electrophysiol 2003;8:27-36.

3. Solomon SB, White P, Wiener CM, Orens JB, Wang KP. Three-dimensional CT-guided bronchoscopy with a real-time electromagnetic position sensor. Chest 2000;118:1783-1787. [PubMed: 11115473]

4. Solomon SB, Magee CA, Acker DE, Venbrux AC. Experimental nonfluoroscopic placement of inferior vena cava filters: Use of an electromagnetic navigation system with previous CT data. J Vasc Interv Radiol 1999;10:92-95. [PubMed: 10872496]

5. Ben-Haim SA, Osadchy D, Schuster I, Gepstein L, Hayam G, Josephson ME. Nonfluoroscopic, in vivo navigation and mapping technology. Nature Medicine 1996;2(12):1393-1395.

6. Wood BJ, Ramkaransingh JR, Fojo T, Walther MM, Libutti SK. Percutaneous tumor ablation with radiofrequency. Cancer 2002;94(2):443-451. [PubMed: 11900230]

7. Krücker, J.; Viswanathan, A.; Borgert, J.; Glossop, N.; Yang, Y.; Wood, BJ. An electro-magnetically tracked laparoscopic ultra-sound for multi-modality minimally invasive surgery. In: Lemke, HU.; Inamura, K.; Doi, K.; Vannier, MW.; Farman, AG., editors. Computer Assisted Radiology and Surgery; Proceedings of the 19th International Congress and Exhibition (CARS 2005); Berlin, Germany. Amsterdam: Elsevier. June 2005; p. 746-751.2005

8. Leyendecker JR, Dodd GD III. Minimally invasive techniques for the treatment of liver tumors. Seminars in Liver Disease 2001;21(2):283-291. [PubMed: 11436578]

9. Ramsey CR, Scaperoth D, Arwood D, Oliver AL. Clinical efficacy of respiratory gated conformal radiation therapy. Med Dosim 1999;24:115-119. [PubMed: 10379508]

10. Korin HW, Ehman RL, Riederer SJ, Felmlee JP, Grimm RC. Respiratory kinematics of the upper abdominal organs: a quantitative study. Magn Reson Med 1992;23:172-178. [PubMed: 1531152]

11. Kubo HD, Hill BC. Respiration gated radiotherapy treatment: a technical study. Phys Med Biol 1996;41:83-91. [PubMed: 8685260]

12. Arellano RS, Maher M, Gervais DA, Hahn PF, Mueller PR. The difficult biopsy: let's make it easier. Curr Probl Diagn Radiol 2003;32(5):218-226. [PubMed: 12963868]

13. Silverman SG, Truncali K, Adams DF, Nawfel MD, Zou KH, Judy PF. CT fluoroscopy-guided abdominal interventions: techniques, results, and radiation exposure. Radiology 1999;212:673-681. [PubMed: 10478231] 
14. Nawfel RD, Judy PF, Silverman SG, Hooton S, Tuncali K, Adams DF. Patient and personnel exposure during CT fluoroscopy-guided interventional procedures. Radiology 2000;216:180-184. [PubMed: 10887246]

15. Gianfelice D, Lepanto L, Perrault P. Value of CT fluoroscopy for percutaneous biopsy procedures. J Vasc Interv Radiol 2000;11:879-884. [PubMed: 10928526]

16. Kato R, Katada K, Anno H, Suzuki S, Ida Y, Koga S. Radiation dosimetry at CT fluoroscopy: physician's hand dose and development of needle holders. Radiology 1996;201:576-578. [PubMed: 8888264]

17. Fröhlich H, Döhring W. A simple device for breath-level monitoring during CT. Radiology 1985;156 (1):235. [PubMed: 4001413]

18. Robinson PJ, Kreel L. Pulmonary tissue attenuation with computed tomography: comparison of inspiration and expiration scans. J Comput Assist Tomogr 1979;3(6):740-748. [PubMed: 512106]

19. Jones KR. A respiration monitor for use with CT body scanning and other imaging techniques. Br $\mathrm{J}$ Radiol 1982;55(655):530-533. [PubMed: 7150902]

20. Robinson PJ, Jones KR. Improved control of respiration during computed tomography by feedback monitoring. J Comput Assist Tomogr 1982;6(4):802-806. [PubMed: 7119202]

21. Berson AM, Emery R, Rodriguez L, Richards GM, Ng T, Sanghavi S, Barsa J. Clinical experience using respiratory gated radiation therapy: comparison of free-breathing and breath-hold techniques. Int J Radiat Oncol Biol Phys 2004;60(2):419-426. [PubMed: 15380575]

22. Timinger H, Krüger S, Dietmayer K, Borgert J. Motion compensated coronary interventional navigation by means of diaphragm tracking and elastic motion models. Phys Med Biol 2005;50(3): 491-503. [PubMed: 15773725]

23. Dietrich L, Tucking T, Nill S, Oelfke U. Compensation for respiratory motion by gated radiotherapy: an experimental study. Phys Med Biol 2005;50(10):2405-2414. [PubMed: 15876675]

24. Timinger, H.; Krüger, S.; Dietmayer, K.; Borgert, J. Motion compensation for interventional navigation on 3D static roadmaps based on a dynamic motion model. In: Lemke, HU.; Vannier, MW.; Inamura, K.; Farman, AG.; Doi, K.; Reiber, JHC., editors. Computer Assisted Radiology and Surgery; Proceedings of the 18th International Congress and Exhibition (CARS 2004); Chicago, IL. Amsterdam: Elsevier. June 2004; 2004; p. 1055-1060.

25. Timinger H, Krüger S, Dietmayer K, Borgert J. Motion compensated coronary interventional navigation by means of diaphragm tracking and elastic motion models. Phys Med and Biol 2005;50 (3):491-503. [PubMed: 15773725]

26. Hardy RL. Multiple equations of topography and other irregular surfaces. J Geophysical Res 1971;76 (8):1905-1915. 

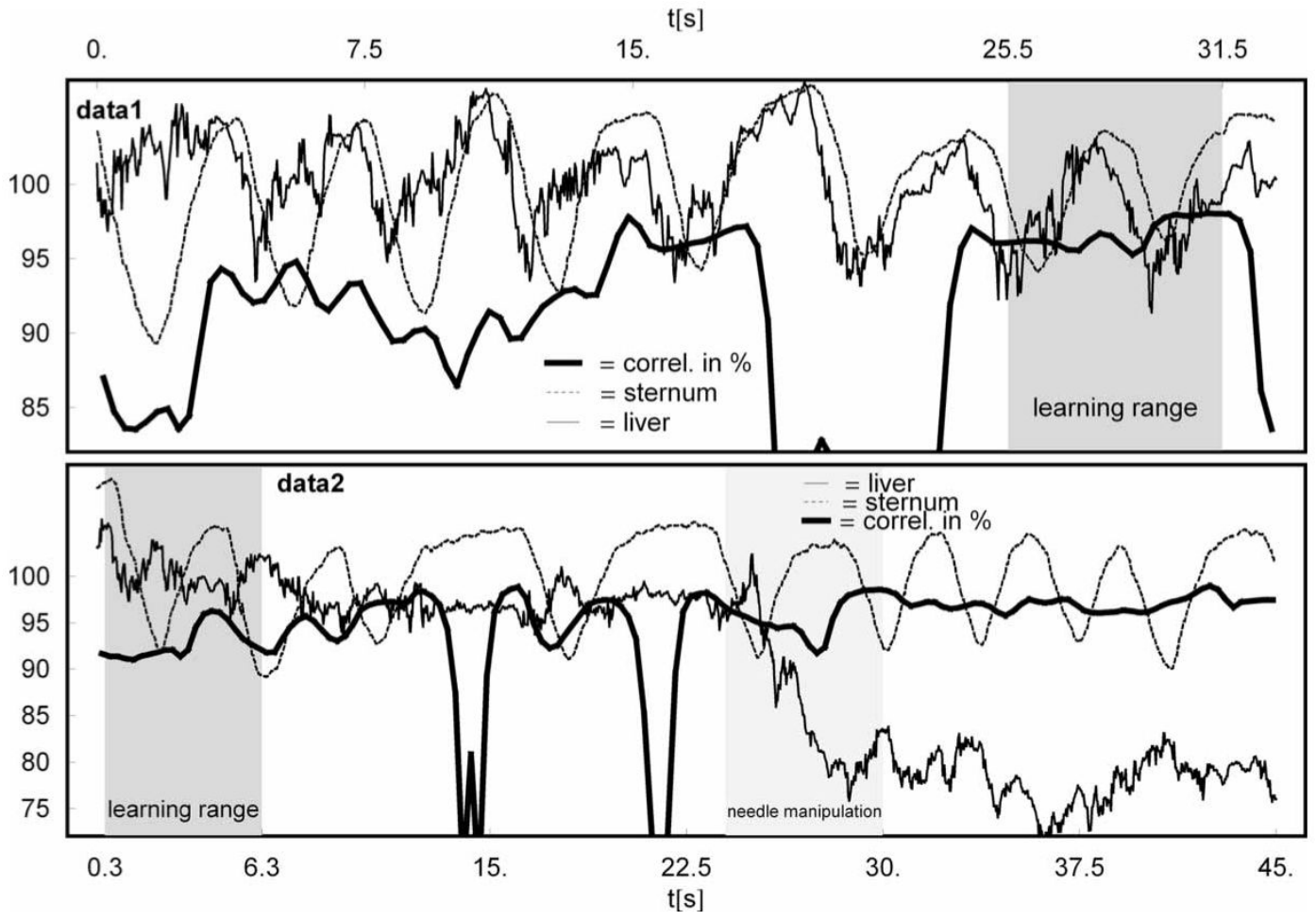

Figure 1.

Absolute positions of liver (thin solid line) and sternum (thin broken line) sensors together with correlation for datal (top) and data2 (bottom). Liver and sternum sensor positions are displayed in arbitrary scaling, whereas correlation is displayed as percentage. 

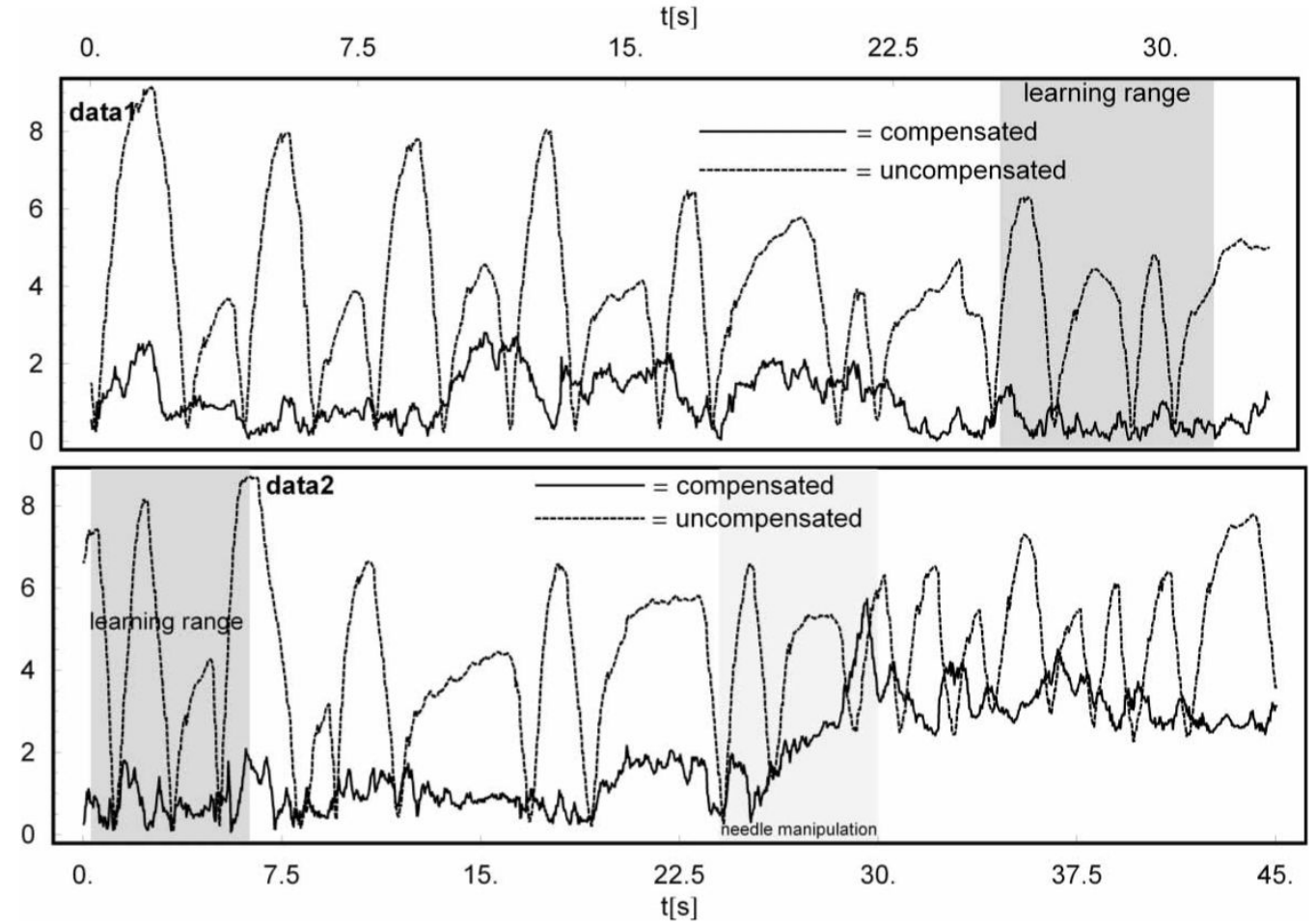

Figure 2.

Uncompensated (broken line) and compensated (solid line) residual displacement in $\mathrm{mm}$ for the needle placed in the liver for datal (top) and data2 (bottom). 
Table I

Characteristics of the selected ranges of data with steady respiration

\begin{tabular}{cccc}
\hline & Duration (s) & No. of samples & No. of respiratory cycles \\
\hline data1 & $\sim 34$ & 1100 & $\sim 9$ \\
data2 & $\sim 46$ & 1500 & $\sim 10$ \\
\hline
\end{tabular}

Needle manipulation between 24.5 and 30.5 seconds in data 2 
Table II

Residual displacement after affine motion compensation. Results from a free-form model for comparison

\begin{tabular}{llc}
\hline Learned from & Applied to & Residual displacement (mm) \\
\hline$\sim 6$ sec. of datal & $\begin{array}{l}\text { all of data1 } \\
\text { (free form model) }\end{array}$ & $0.99 \pm 0.66$ \\
& $\begin{array}{l}\text { first 23 s of data2 } \\
\text { last 15 s of data2 } \\
\text { (free form model - all of } \\
\text { data2) }\end{array}$ & $0.94 \pm 0.91)$ \\
& & $3.18 \pm 0.48$ \\
& & $(1.99 \pm 1.09)$ \\
$\begin{array}{l}\text { Displacement of data1 } \\
\text { without compensation }\end{array}$ & & $3.90 \pm 2.00$ \\
$\begin{array}{l}\text { Displacement of data2 } \\
\text { without compensation }\end{array}$ & & $4.18 \pm 2.10$ \\
\hline
\end{tabular}

